\title{
Die Rolle der Parlamente beim Auslandseinsatz deutscher und britischer Streitkräfte*
}

\section{Johannes Hinz}

Aus verfassungsrechtlicher Sicht lässt sich die Entscheidung über den Einsatz von Streitkräften in zwei Bereiche kategorisieren: die generelle Zulässigkeit von Auslandseinsätzen nach der Verfassung und die Entscheidungskompetenz der Verfassungsorgane, insbesondere die Einbeziehung des Parlaments. ${ }^{1}$ Letztere berührt grundlegende Prinzipien der staatlichen Ordnung, wirft aber auch praktische Fragen nach Form, Reichweite, Durchsetzung, Verfahren und Grenzen der Beteiligung auf. Diese Fragen bewegen sich in einem Spannungsfeld zwischen demokratischer Kontrolle der Exekutive sowie Transparenz einerseits und Schnelligkeit, Effektivität sowie Sicherheit der militärischen Operationen andererseits ${ }^{2}$ : Eine Einbeziehung des Parlaments in konkrete Einsatzentscheidungen scheint daher zwangsläufig eine Einschränkung der Effektivität dieser Operationen zu bedeuten. Jeder demokratische Staat muss also ein Gleichgewicht zwischen diesen gegensätzlichen Positionen herstellen.

In Fragen der Streitkräfte verhalten sich wohl kaum zwei andere einflussreiche westliche Staaten so unterschiedlich wie die Bundesrepublik Deutschland und das Vereinte Königreich. Großbritannien hat zwischen 1946 und 2003 an mehr internationalen Konflikten teilgenommen als jeder andere Staat der Welt. ${ }^{3}$ Dementsprechend hat es auch einen im Verhältnis zum Bruttosozialprodukt circa 70 Prozent größeren Verteidigungsetat als Deutschland ${ }^{4}$ und gibt pro Kopf fast doppelt so viel für Verteidigung aus. ${ }^{5}$ Es liegt nahe, dass sich diese Unterschiede auch im Recht und insbesondere in der Kontrolle der Streitkräfteeinsätze abbilden. Ein Vergleich zu Deutschland bietet sich zudem an, weil sich Großbritannien derzeit in einer Phase der kritischen Reflexion über die eigene Verfassung befindet, in der die "genuinely constitutional blockbuster issues" 6 diskutiert werden und die auch auf dem Gebiet der „war powers“ zu einiger Bewegung geführt hat.

* Der Beitrag basiert auf der Magisterarbeit des Verfassers, die im Wintersemester 2010/2011 an der Juristischen Fakultät der Leibniz Universität Hannover vorgelegt wurde.

1 Vgl. Volker Epping, Die Evakuierung deutscher Staatsbürger im Ausland als neues Kapitel der Bundeswehrgeschichte ohne rechtliche Grundlage? Der Tirana-Einsatz der Bundeswehr auf dem rechtlichen Prüfstand, in: Archiv des öffentlichen Rechts, 124. Jg. (1999), H. 3, S. 423 - 469, S. 426.

2 Vgl. David Jenkins, Efficiency and Accountability in War Powers Reform, in: Journal of Conflict and Security Law, 14. Jg. (2009), H. 1, S. 145 - 165, S. 148; Wolfgang Weiß, Die Beteiligung des Bundestags bei Einsätzen der Bundeswehr im Ausland - eine kritische Würdigung des Parlamentsbeteiligungsgesetzes, in: Neue Zeitschrift für Wehrrecht, 46. Jg. (2005), H. 3, S. 100 - 116, S. 102.

3 Vgl. Human Security Centre, Human Security Report 2005, New York / Oxford 2005, S. 26 f.; John Van Wingen / Herbert Tillema, British Military Intervention after World War II: Militance in a Second-Rank Power, in: Journal of Peace Research, 17. Jg. (1980), H. 4, S. 291 - 303, S. 291.

4 Vgl. MOD/DASA, UK Defence Statistics, Table 1.20, http://www.dasa.mod.uk/modintranet/ UKDS/UKDS2009/pdf/UKDS2009.pdf (Abruf am 28. Mai 2011).

5 Vgl. ebenda, Table 1.21.

6 Andrew Le Sueur, Gordon Brown's New Constitutional Settlement, in: Public Law, 52. Jg. (2008), H. 1, S. 21 - 27, S. 21. Dazu gehören etwa die Besetzung und Stellung des House of Lords, quasi-richterliche Befugnisse von Mitgliedern der Exekutive, die Ausweitung gerichtlicher Kontrolle durch den Human Rights Act 1998 und die Ernennung von Richtern. 
Die Entstehung des deutschen Parlamentsvorbehalts ist Gegenstand umfassender wissenschaftlicher Untersuchung. ${ }^{7}$ Zur Erinnerung sei hier erwähnt, dass das Bundesverfassungsgericht ihn im Jahr 1994 entgegen der wohl überwiegenden Ansicht in der Literatur ${ }^{8}$ entwickelte und den Gesetzgeber aufforderte, ihn auszugestalten. ${ }^{9}$ Dies geschah 2005 durch das Parlamentsbeteiligungsgesetz (ParlBG).

\section{Die Rechtslage in Großbritannien}

\subsection{War Powers in der britischen Verfassung}

Die britische Verfassung reicht in manchen Grundstrukturen bis ins 13. Jahrhundert zurück. ${ }^{10}$ Sie ist insofern ungewöhnlich, als sie im Gegensatz etwa zur deutschen Verfassung nicht kodifiziert ${ }^{11}$ und rechtlich nicht besonders gegen Änderungen geschützt (,entrenched") ist ${ }^{12}$, wodurch sie ständigem Wandel unterworfen ist. ${ }^{13}$ Eine verbreitete Ansicht hält eine kodifizierte Verfassung für zu unflexibel, stattdessen seien politische „checks and balances" eingesetzt. ${ }^{14}$ Im Gegensatz zur Technik der Kodifikation besteht die britische Verfassung aus einer Vielzahl verschiedener Quellen: Gesetze und Verordnungen enthalten eben-

7 Vgl. Rafael Biermann, Der Deutsche Bundestag und die Auslandseinsätze der Bundeswehr. Zur Gratwanderung zwischen exekutiver Prärogative und legislativer Mitwirkung, in: ZParl, 35. Jg. (2004), H. 4, S. 607 - 626; Dieter Wiefelspütz, Der konstitutive wehrverfassungsrechtliche Parlamentsbeschluss, in: ZParl, 38. Jg. (2007), H. 1, S. 3 - 15; ders., Auslandseinsätze deutscher Streitkräfte und der Bundestag: Ist eine Reform geboten?, in: ZParl, 39. Jg. (2008), H. 2, S. 203 - 218; für einen Überblick Phillip Scherrer, Das Parlament und sein Heer: Das Parlamentsbeteiligungsgesetz, Berlin 2010.

8 Vgl. Knut Ipsen, Bündnisfall und Verteidigungsfall, in: Die Öffentliche Verwaltung, 24. Jg. (1971), H. 14, S. 585 - 588, S. 587; Klaus Kersting, Die Entscheidung über den Einsatz der Bundeswehr zur Verteidigung, in: Neue Zeitschrift für Wehrrecht, 23. Jg. (1982), H. 3, S. $84-$ 91, S. 86; Norbert Riedel, Der Einsatz deutscher Streitkräfte im Ausland, Frankfurt am Main 1989, S. 248 ff.; Rupert Scholz, Stand und Perspektiven der Verfassungsdiskussion in der Bundesrepublik Deutschland, in: Jürgen Schwarz / Armin Steinkamm, Rechtliche und politische Probleme des Einsatzes der Bundeswehr „out of area”, Baden-Baden 1993, S. 29 - 41, S. 39; Mark Zimmer, Einsätze der Bundeswehr im Rahmen kollektiver Sicherheit, Frankfurt am Main 1995, S. 142 ff.; Manfred Lepper, Die verfassungsrechtliche Stellung der militärischen Streitkräfte im gewaltenteilenden Rechtsstaat, Bielefeld 1962, S. 94 ff., S. 157; Marcus Schultz, Die Auslandsentsendung von Bundeswehr und Bundesgrenzschutz, Frankfurt am Main 1998, S. 431.

9 Vgl. BVerfGE 90, S. 286 (S. 381, S. 389).

10 Vgl. Colin Munro, Studies in Constitutional Law, Oxford 1999, S. 260 f. Auch heute noch von Bedeutung ist etwa die Magna Charta von 1215.

11 Vgl. Anthony Lester / Matthew Weait, The Use of Ministerial Powers Without Parliamentary Authority: The Ram Doctrine, in: Public Law, 47. Jg. (2003), H. 3, S. 415 - 428, S. 418; Colin Turpin / Adam Tomkins, British Government and the Constitution, Cambridge 2007, S. 139 weisen in diesem Zusammenhang darauf hin, dass das oft benutzte Etikett „ungeschrieben” nicht zutrifft, da nahezu alle Regeln schriftlich niedergelegt sind.

12 Vgl. Colin Turpin / Adam Tomkins, a.a.O. (Fn. 11), S. 6 f.

13 Vgl. Select Committee on the Constitution, Waging War: Report, London 2006, S. 11.

14 Vgl. Iain Byrne / Stuart Weir, Democratic Audit: Executive Democracy in War and Peace, in: Parliamentary Affairs, 57. Jg. (2004), H. 2, S. 453 - 468, S. 453. 
so Prinzipien von Verfassungscharakter wie das Common Law und Konventionen ${ }^{15}$ Kritiker sprechen insofern von einer „ramshackle constitution” ${ }^{16}$. Konventionen sind Regeln, die als verbindlich anerkannt die Beziehungen zwischen den Teilen der Verfassung regeln, aber nicht durch die Gerichte durchgesetzt werden. ${ }^{17}$ Ein Verstoß hat lediglich politische Konsequenzen. ${ }^{18}$ Konventionen können durch Gesetz anerkannt, formuliert oder vorausgesetzt ${ }^{19}$ und durch regelmäßige Nichtbefolgung aufgelöst werden. ${ }^{20}$ Gerade in hochpolitischen Bereichen, die als rechtlich schwer zu erfassen gelten, sind sie von großer Bedeutung. ${ }^{21}$ Ebenso vielfältig sind die Quellen exekutiver Befugnisse: „The legal sources of executive powers are derived from statutes, and, more obscurely, from the royal prerogative, and common law powers claimed as being derived from the legal personality of the crown." 22

Wesentliche Prinzipien der britischen Verfassung sind die Gewaltenteilung ${ }^{23}$, die aber vornehmlich als exklusive Zuweisung von Funktionen unter Ausschluss von Kontrolle verstanden wird ${ }^{24}$, sowie die „rule of law" mit der Ausprägung des Grundsatzes der „legality“, die eine Ermächtigungsgrundlage für staatliches Handeln fordert. ${ }^{25}$ Ein weiteres wesentliches Prinzip ist das der „parliamentary sovereignty”. Bereits bei John Locke findet sich die Feststellung: „There can be but one Supreme Power, which is the Legislative, to which all the rest are and must be subordinate."26 Die Etablierung des Vorrangs des Parlaments war ein wesentlicher Teil des Revolution Settlement aus dem Jahr 168827: Dabei geht das Parlamentsgesetz (,statute") allen anderen nationalen und internationalen Rechtsquellen vor. ${ }^{28}$

Vielfach für problematisch gehalten wird aber die starke Stellung der Regierung im Parlament. Zwar kann das Parlament ihr das Vertrauen entziehen ${ }^{29}$, jedoch ist dies eher eine theoretische Möglichkeit: Durch die Befugnis des Premierministers, die Auflösung des Par-

15 Vgl. dazu etwa Colin Turpin / Adam Tomkins, a.a.O. (Fn. 11), S. 138 ff.

16 Thomas Poole, The Royal Prerogative, in: International Journal of Constitutional Law, 8. Jg. (2010), H. 1, S. 146 - 155, S. 147.

17 Vgl. Geoffrey Marshall, Constitutional Conventions, Oxford 1984, S. 210 ff.; O. Hood Phillips I Paul Jackson, O. Hood Phillips' Constitutional and Administrative Law, London 1987, S. 113; Colin Turpin / Adam Tomkins, a.a.O. (Fn. 11), S. 156 f.; Lord Wilson of Dinton, The Robustness of Conventions in a Time of Modernisation and Change, in: Public Law, 48. Jg. (2004), H. 2, S. 407, S. $408 \mathrm{f}$.

18 Vgl. O. Hood Phillips / Paul Jackson, a.a.O. (Fn. 17), S. 117; Lord Wilson of Dinton, a.a.O. (Fn. 17), S. 407 - 420, S. 410.

19 Vgl. O. Hood Phillips / Paul Jackson, a.a.O. (Fn. 17), S. 118.

20 Vgl. ebenda, S. 121.

21 Vgl. Basil Markensis, The Royal Prerogative re-visited, in: Cambridge Law Journal, 32. Jg. (1973), H. 2, S. $287-309$, S. 307.

22 Anthony Lester / Matthew Weait, a.a.O. (Fn. 11), S. 418.

23 Vgl. Lord Diplock, in: Dupont Steels Ltd. v Sirs et al. [1980] 1 WLR 142 (157); Colin Turpin I Adam Tomkins, a.a.O. (Fn. 11), S. 103 ff.

24 Vgl. Lord Lester of Herne Hill, in: Select Committee on the Constitution, Waging War: Evidence, London 2006, S. 16.

25 Vgl. Anthony Lester / Matthew Weait, a.a.O. (Fn. 11), S. 419.

26 Vgl. John Locke, Two Treatises of Government, London 1698, S. $281 \mathrm{ff}$.

27 Vgl. Colin Munro, a.a.O. (Fn. 10), S. 271.

28 Vgl. Colin Turpin / Adam Tomkins, a.a.O. (Fn. 11), S. 40.

29 Vgl. Julian Samiloff, Who Should Declare War?, in: New Law Journal, 156. Jg. (2008), H. 7318, S. 565 . 
laments zu bewirken, sowie die starke Stellung, die das Mehrheitswahlrecht der Regierung einräumt, besteht nach verbreiteter Ansicht keine effektive Trennung im Sinne von checks and balances zwischen Regierung und Parlament. ${ }^{30}$

Von besonderer Bedeutung für die Organkompetenz zur Entscheidung über einen Einsatz von Streitkräften ist die „royal prerogative“, eine Rechtsquelle, die bereits zu Beginn des 20. Jahrhunderts als „extravagantly archaic” bezeichnet ${ }^{31}$ wurde und auch gegenwärtig vielfach als unzeitgemäß empfunden wird. ${ }^{32}$ Ihren Ursprung hat sie in der Einheit von Staat und Monarch: Die bis heute verbliebenen Kompetenzen sind die Bereiche der vormals umfassenden Macht des Monarchen, die ihm nicht durch das Parlament entzogen wurden. ${ }^{33}$ Dabei sind wesentliche ${ }^{34}$ verfassungsrechtliche Weichenstellungen wie etwa die Einberufung und Auflösung des Parlaments, die Gesetzgebung durch „Orders in Council“, die Begnadigung von Straftätern, der Abschluss völkerrechtlicher Verträge, das Passwesen, die Verleihung von Ehren, die Ernennung des Premierministers und die Verkündung von Gesetzen ${ }^{35}$ ebenso Teil der königlichen Prärogative wie sehr spezielle Bereiche, etwa das Recht der Krone auf sämtliche Störe, Schwäne und Wale sowie auf den Abbau von Salpeter und die Verwertung von Schiffswracks. ${ }^{36}$

Nach einer Konvention ${ }^{37}$ werden die Rechte und Ermächtigungen inzwischen nahezu vollständig ${ }^{38}$ durch Minister oder auf Anraten von Ministern ausgeübt. Ursprung dieser Verschiebung war die immer breiter werdende demokratische Legitimation des Premierministers mit Ausbildung von politischen Parteien, so dass es unter politischen Gesichtspunkten geboten schien, diesem die Entscheidungen zu überlassen. ${ }^{39}$ Damit ging allerdings auch eine teilweise Abnahme demokratischer Kontrolle einher: Während dem König ein Par-

30 Vgl. Eric Barendt, Separation of Powers and Constitutional Government, in: Public Law, 49. Jg. (1995), H. 4, S. 599 - 619, S. 613 f.; Hilaire Barnett, Constitutional \& Administrative Law, London 2004, S. 154 f.; David Jenkins, a.a.O. (Fn. 2), S. 160; Lord Wilson of Dinton, a.a.O. (Fn. 17), S. 415.

31 Vgl. Vivian St. Clair Mackenzie, The Royal Prerogative in War-Time, in: The Law Quarterly Review, 34. Jg. (1918), H. 134, S. 152 - 159, S. 157.

32 Vgl. Roger Smith, Rights \& Wrongs: The Roads to War, in: Law Society Gazette, 105. Jg. (2008), H. 12, S. 9; ähnlich David Dyzenhaus, Intimations of Legality Amid the Clash of Arms, in: International Journal of Constitutional Law, 2. Jg. (2004), H. 2, S. 244 - 271, S. 246.

33 Vgl. John Burgess, Political Science and Comparative Constitutional Law, Boston 1891, S. 198; Colin Munro, a.a.O. (Fn. 10), S. 256 f.; Thomas Poole, a.a.O. (Fn. 16), S. 146.

34 Einen der deutschen Wesentlichkeitstheorie entsprechenden Rechtssatz, der ein formelles Gesetz für wichtige Bereiche verlangen würde, gibt es in Großbritannien nicht; vorsichtige Vorschläge in Richtung eines Gesetzesvorbehalts für grundrechtsrelevante und sonstige wichtige Gebiete finden sich aber bei Margit Cohn, Judicial Review of Non-Statutory Executive Powers after Bancoult: A Unified Anxious Model, in: Public Law, 53. Jg. (2009), H. 2, S. 260 - 286, S. 274 ff.

35 Vgl. Lucinda Maer / Gay Oonagh, The Royal Prerogative, House of Commons Standard Note SN/PC/03861, http://www.parliament.uk/documents/commons/lib/research/briefings/snpc03861.pdf (Abruf am 28. Mai 2011), S. 4 f.

36 Die wohl umfassendste Auflistung findet sich in Secretary of State for Justice, The Governance of Britain: Review of the Executive Royal Prerogative Powers: Final Report, London 2009, S. $31 \mathrm{ff}$.

37 Vgl. O. Hood Phillips / Paul Jackson, a.a.O. (Fn. 17), S. 124.

38 Vgl. Helen Fenwick / Gavin Phillipson, Public Law \& Human Rights, Oxon 2003, S. 480; Colin Munro, a.a.O. (Fn. 10), S. 268 f.; Thomas Poole, a.a.O. (Fn. 16), S. 146.

39 Vgl. Sebastian Payne, The Royal Prerogative, in: Maurice Sunkin / ders., The Nature of the Crown: A Legal and Political Analysis, Oxford 1999, S. 77 - 110, S. 100. 
lament mit der „power of the purse“ gegenüberstand, weiß der Premierminister die Mehrheit der Commons hinter sich. ${ }^{40}$

Nachdem die Streitkräfte wesentlicher Streitpunkt der Glorious Revolution waren, wurde 1689 durch die Bill of Rights die Unterhaltung einer Armee zu Friedenszeiten generell unter Parlamentsvorbehalt gestellt. ${ }^{41} \mathrm{Im}$ Bereich der königlichen Prärogative verblieben aber Einsatz und Organisation der Streitkräfte ${ }^{42}$ - damit auch eine exklusive Befugnis zur Erklärung und Führung von Kriegen. ${ }^{43}$ Diese bedarf rechtlich auch keinerlei Beteiligung des Parlaments. ${ }^{44}$ Die grundlegende Überlegung findet sich bereits bei John Locke: Da Gesetze nicht jeden Fall vorhersehen könnten, müsse die Exekutive zum Schutz der Gesellschaft flexibel handeln können. ${ }^{45}$ Tragende Erwägung ist also eine Art Notstandsbefugnis („necessity“). ${ }^{46}$ Die Ausübung der Befugnisse in diesem Bereich obliegt dem Premierminister. ${ }^{47}$

40 Vgl. Select Committee on the Constitution, a.a.O. (Fn. 13), S. 18.

41 „[T] he raising or keeping a standing army within the kingdom in time of peace, unless it be with consent of Parliament, is against law", http://avalon.law.yale.edu/17th_century/england.asp (Abruf am 28. Mai 2011); siehe auch David Jenkins, Constitutional Reform Goes to War: Some Lessons from the United States, in: Public Law, 51. Jg. (2007), H. 2, S. 258 - 279, S. 260; Colin Munro, a.a.O. (Fn. 10), S. 266; Select Committee on the Constitution, a.a.O. (Fn. 13), S. 4 f.; Peter Rowe, The Crown and Accountability for the Armed Forces, in: Maurice Sunkin / Sebastian Payne, a.a.O. (Fn. 39), S. 267 - 282, S. 268.

42 Vgl. LJ Scrutton, China Navigation Company Ltd. v Attorney-General [1932] 2 KB 197 (228).

43 Vgl. Hilaire Barnett, a.a.O. (Fn. 30), S. 131; John Burgess, a.a.O. (Fn. 33), S. 202 ff.; Rodney Brazier, in: PASC (Public Administration Select Committee), Taming the Prerogative, London 2004, S. 25; Helen Fenwick / Gavin Phillipson, a.a.O. (Fn. 38), S. 482 f.; Samuel Issacharoff, Political Safeguards in Democracies at War, in: Oxford Journal of Legal Studies, 29. Jg. (2009), H. 2, S. 189 - 214, S. 201; Colin Munro, a.a.O. (Fn. 10), S. 258; O. Hood Phillips / Paul Jackson, a.a.O. (Fn. 17), S. 345; Lord Reid, in: Chandler v DPP [1964] AC 763 (791); Peter Rowe, a.a.O. (Fn. 41), S. 270; Julian Samiloff, a.a.O. (Fn. 29), S. 565; Claire Taylor / Richard Kelly, Parliamentary Approval for Deploying the Armed Forces, Research Paper 08/88, London 2008, S. 8; Francis Canon Tudsberry, Prerogative in Time of War, in: The Law Quarterly Review, 32. Jg. (1916), H. 128, S. 384 - 391, S. 384; Colin Turpin / Adam Tomkins, a.a.O. (Fn. 11), S. 163; Colin Warbrick, The Governance of Britain, in: International and Comparative Law Quarterly, 57. Jg. (2008), H. 1, S. 209 - 217, S. 213; Nigel White, The United Kingdom: Increasing Commitment Requires Greater Parliamentary Involvement, in: Charlotte Ku / Harold Jacobson, Democratic Accountability and the Use of Force in International Law, Cambridge 2003, S. 300 - 322, S. 300; David Yale, Sir Matthew Hale's The Prerogatives of the King, London 1976, S. 173; bereits N.N., A Vindication of the Prerogative of the Crown, London 1703, S. 9; John Morphew, The Honour and Prerogative of the Queen's Majesty Vindicated and Defended, London 1713, S. 14. Eine andere Ansicht findet sich vereinzelt im 17. und frühen 18. Jahrhundert, so etwa mit naturrechtlichen Argumenten Thomas Underhill, Touching the Fundamentall Lawes, London 1642, S. 10 f.; unter Verweis auf die große Wichtigkeit dieser Frage Charles Davenant, zitiert nach A. Baldwin, The Old and New Ministry Compar'd \& c., London 1711, S. 13 ff., S. 38. Die Bezeichnung Krieg wurde/wird dabei in der englischsprachigen Literatur zumeist nicht im völkerrechtlichtechnischen, sondern im umgangssprachlichen Sinne gebraucht.

44 Vgl. Andrew Blick / Iain Byrne / Stuart Weir, Democratic Audit: Good Governance, Human Rights, War Against Terror, in: Parliamentary Affairs, 58. Jg. (2005), H. 2, S. 408 - 423, S. 409; Iain Byrne / Stuart Weir, a.a.O. (Fn. 14), S. 454; Samuel Issacharoff, a.a.O. (Fn. 43), S. 203 f.; Claire Taylor I Richard Kelly, Parliamentary Approval for Deploying the Armed Forces, a.a.O. (Fn. 43), S. 8.

45 Vgl. John Locke, a.a.O. (Fn. 26), S. 291; ähnlich Francis Canon Tudsberry, a.a.O. (Fn. 43), S. $388 \mathrm{ff}$.

46 Vgl. Sebastian Payne, a.a.O. (Fn. 39), S. 92.

47 Vgl. David Jenkins, a.a.O. (Fn. 41), S. 263; Julian Samiloff, a.a.O. (Fn. 29), S. 565. 
Zwar stehen dem Parlament allgemeine Kontrollmechanismen wie Fragen ${ }^{48}$ und Diskussionen zur Verfügung, jedoch nur ex post ${ }^{49}$ und unter besonderen Schwierigkeiten: Die Regierung sieht sich teilweise unter Verweis auf die Prärogative nicht in der Pflicht, Fragen zu beantworten. ${ }^{50}$ Entgegen der förmlichen Nichtzuständigkeit des Parlaments wurden bisher viele größere Operationen nach Beginn im Parlament diskutiert und einer (deklaratorischen) Abstimmung unterzogen. ${ }^{51}$

Nach klassischer Ansicht war die Ausübung der königlichen Prärogative nicht gerichtlich überprüfbar. ${ }^{52}$ Dazu John Locke: „But who shall be Judge when this Power is made right use of? [...] there can be no Judge on Earth. [...] The People have no other remedy in this [...] but to appeal to Heaven." 53 Einen nächsten Schritt stellte insofern das Urteil des House of Lords im Fall „Council of Civil Service Unions et al. versus Minister for the Civil Service" 54 dar. Danach sei auch die Ausübung der Prärogative grundsätzlich gerichtlich überprüfbar ${ }^{55}$, soweit justiziabel: Dies sei aber die Verteidigung nicht. ${ }^{56}$ Entsprechend ist die Judikatur in diesem Bereich fast ausnahmslos von Zurückhaltung geprägt, soweit politisch aufgeladene Fragen betroffen sind. ${ }^{57}$ Damit ist derzeit die Entscheidung über den Einsatz von Truppen in Großbritannien nicht überprüfbar ${ }^{58}$, sondern wird als „area of policy" ${ }^{59}$ und exklusiver Verantwortungsbereich der Exekutive aufgefasst. ${ }^{60}$ Die dem zugrunde liegende Argumentation deckt sich mit der klassischen Begründung gegen eine Parlamentsbeteiligung: „Those responsible for national security must be the sole judges of what the national security requires. It would be obviously undesirable that such matters should be made the subject of evidence in a Court of law or otherwise discussed in public." ${ }^{1}$ Es gibt allerdings Hinweise darauf, dass die Gerichte bei einer gesetzlichen Grundlage, die ei-

48 Vgl. Peter Rowe, a.a.O. (Fn. 41), S. 273.

49 Vgl. Colin Munro, a.a.O. (Fn. 10), S. 277. Zu den dem Parlament zur Verfügung stehenden (politischen) Mitteln der Kontrolle siehe Colin Turpin / Adam Tomkins, a.a.O. (Fn. 11), S. 606 ff.

50 Vgl. Colin Munro, a.a.O. (Fn. 10), S. 277.

51 Vgl. Roger Smith, a.a.O. (Fn. 32), S. 9. Eine Ausnahme davon ist aber etwa der sehr umfangreiche Einsatz im Rahmen der ISAF, vgl. Claire Taylor / Richard Kelly, a.a.O. (Fn. 43), S. 13 f.

52 Vgl. Helen Fenwick / Gavin Phillipson, a.a.O. (Fn. 38), S. 494; Colin Turpin / Adam Tomkins, a.a.O. (Fn. 11), S. 696.

53 John Locke, a.a.O. (Fn. 26), S. 297.

54 Council of Civil Service Unions et al. v Minister for the Civil Service [1985] AC 374.

55 Vgl. Lord Scarman / Lord Roskill, in: ebenda (407, 417).

56 Vgl. Lord Roskill, in: ebenda (418). Gegen das Argument des fehlenden rechtlichen Maßstabs führt Colin Warbrick, a.a.O. (Fn. 43), S. 212 f., an, dass das internationale Recht einen Maßstab biete, aber grundsätzlich nicht unmittelbar anwendbar ist.

57 Vgl. David Dyzenhaus, a.a.O. (Fn. 32), S. 246. Dem stehen Forderungen gegenüber, „judicial review" auch auf diese Bereiche auszuweiten; dies sei der nächste logische Schritt in dessen Entwicklung, vgl. Brian Marinelli, United States and United Kingdom: Evaluating the Judicial Review of the Government's Power to Declare War, in: Suffolk Transnational Law Review, 21. Jg. (1997/1998), H. 2, S. 473 - 487, S. 487.

58 Vgl. Lord Parker of Waddington, in: The Zamora [1916] 2 AC 77 (92); LJ Scrutton, a.a.O. (Fn. 42), S. 214; Waller in Ayliffe et al. v Director of Public Prosecutions [2005] 3 WLR 628 (644 und 646); Lord Bingham of Cornhill und Lord Hoffman in R v Jones (Margaret) et al. [2007] 1 AC 136 (162 und 172); David Jenkins, Constitutional reform goes to war: some lessons from the United States, a.a.O. (Fn. 42), S. 267 f.

59 Vgl. Sir Anthony Clarke, in: R (Gentle at al.) v Prime Minister et al. [2007] QB 689 (712).

60 Vgl. ebenda.

61 Vgl. Lord Parker of Waddington, a.a.O. (Fn. 58), S. 107. 
nen Maßstab für die Prüfung der Entscheidung bieten würde, ihre Zurückhaltung ablegen und auch eine als politisch empfundene Entscheidung einer Prüfung unterziehen würden. ${ }^{62}$

\subsection{Entwürfe für eine Reform}

Die oben beschriebene verfassungsrechtliche Position geriet seit 2003 zunehmend in die Kritik. Auslöser war, dass es zur Unterstützung der USA im Krieg gegen den Irak zwar eine parlamentarische Abstimmung gab ${ }^{63}$, diese aber auf einer vielfach als unzureichend empfundenen Informationspraxis der Regierung beruhte. ${ }^{64}$ Demgegenüber hatten vor Amtsantritt spätere Mitglieder der betreffenden Regierung Tony Blairs die Prärogative noch als zu beseitigenden Anachronismus charakterisiert. ${ }^{65}$ Die von diesem Zeitpunkt an zunehmende Forderung nach mehr demokratischer Kontrolle des Einsatzes von Streitkräften hat zu verschiedenen Entwürfen neuer Mechanismen im parlamentarischen Verfahren geführt.

2004 legte ein Ausschuss des House of Commons, das Public Administration Select Committee (PASC), den Bericht „Taming the Prerogative: Strengthening Ministerial Accountability to Parliament" vor. Darin empfahl das PASC, ein Gesetz zu erlassen ${ }^{66}$, für das es auch einen konkreten und recht weitreichenden Entwurf vorlegte ${ }^{67}$, auf den die Regierung wenig enthusiastisch antwortete. ${ }^{68}$

Eine weitere Befassung mit Fragen der Prärogative, speziell mit dem Bereich Streitkräfteeinsatz und Krieg, erfolgte 2006 durch das House of Lords Select Committee on the Constitution (SCC) in seinem Bericht „Waging War. Parliament's Role and Responsibility“.

62 LJ Scrutton, a.a.O. (Fn. 42), S. 214: „This is because the administration of the army is in the hands of the King, who unless expressly controlled by an Act of Parliament cannot be controlled by the Courts." Bereits in Jackson v Attorney General [2005] UKHL 56 hat das Gericht entgegen britischer Tradition das ordnungsgemäße Zustandekommen eines Parlamentsgesetzes geprüft nachdem es dafür konkrete gesetzliche Maßstäbe in Form der Parliament Acts 1911 und 1949 gab, vgl. Colin Turpin / Adam Tomkins, a.a.O. (Fn. 11), S. 46 f.

63 Diese wurde von Tony Blair informell mit einer Art „Vertrauensfrage” verknüpft; Iain Byrne I Stuart Weir, a.a.O. (Fn. 14), S. 455. Einige Kommentatoren sehen in der Beteiligung des Parlaments die Begründung einer Konvention, etwa Rodney Brazier, a.a.O. (Fn. 43), S. 25; jedoch ist es zweifelhaft, ob ein einzelner Präzedenzfall eine Konvention begründen kann und ob dies im vorliegenden Fall geschehen ist; Helen Fenwick / Gavin Phillipson, Public Law \& Human Rights, a.a.O. (Fn. 38), S. 483.

64 Vgl. Samuel Issacharoff, a.a.O. (Fn. 43), S. 202; David Jenkins, a.a.O. (Fn. 2), S. 147 f.; Sebastian Payne, a.a.O. (Fn. 39), S. 24; Colin Warbrick, a.a.O. (Fn. 43), S. 211; zu Details der Informationspraxis und damit verbundenen Skandalen siehe auch Alan Doig, 45 Minutes of Infamy? Hutton, Blair and the Invasion of Iraq, in: Parliamentary Affairs, 58. Jg. (2005), H. 1, S. $109-123$.

65 Vgl. Keith Syrett, Prerogative Powers: New Labour's forgotten constitutional reform?, in: Denning Law Journal, 13. Jg. (1998), S. 111 - 129, S. 117. Auch sonst tut sich bei Vorschlägen zur Reform der Prärogative hauptsächlich die jeweilige Opposition hervor, vgl. PASC, a.a.O. (Fn. 43), S. 8; Claire Taylor / Richard Kelly, a.a.O. (Fn. 43), S. 17 f.

66 Vgl. PASC, a.a.O. (Fn. 43), S. 12 f.

67 Vgl. ebenda, S. 35 f.

68 Vgl. Government Response to the Public Administration Select Committee's Fourth Report of the 2003-04 Session, London 2004, http://www.dca.gov.uk/pubs/reports/prerogative.htm (Abruf am 28. Mai 2011). 
Bezüglich einer stärkeren Rolle des Parlaments wog das SCC Vor- und Nachteile gegeneinander ab: Einerseits garantiere eine größere Rolle des Parlaments mehr Legitimität, mehr Verantwortlichkeit, besser durchdachte Entscheidungen und bei Zustimmung eine bessere Moral der Truppe ${ }^{69}$; andererseits seien ein Effektivitätsverlust und Verzögerung der Entscheidung sowie Informationsdefizite des Parlaments und eine schlechtere Moral der Truppe bei nur knapper Befürwortung eines Einsatzes zu befürchten. ${ }^{70}$ Nach dieser Abwägung kam das SCC zu dem Ergebnis, eine Konvention sei der beste Weg zu mehr Einbeziehung des Parlaments. ${ }^{71}$ Die Antwort der Regierung fiel ähnlich wie zum Bericht des PASC aus: Man sei „not presently persuaded”; Misstrauensvotum, Debatte, Fragen und Ausschüsse böten ausreichende Kontrolle. ${ }^{72}$

Eine Wende in der Position der Regierung fand nach dem Wechsel von Tony Blair zu Gordon Brown 2007 statt. Ein breit angelegter Reformplan unter dem Titel „The Governance of Britain” hatte zum Ziel, Befugnisse, die nicht alleine der Exekutive zustehen sollten zu beschränken oder aufzugeben, so etwa den Streitkräfteeinsatz, die Auflösung des Parlaments und die Ratifikation von Verträgen. ${ }^{73}$ Wichtige Entscheidungen, die das gesamte Land betreffen, sollten nunmehr mit Zustimmung des Parlaments getroffen werden. ${ }^{74}$

In der Frage des Streitkräfteeinsatzes kam das Justizministerium wie die Lords ${ }^{75}$ zu dem Ergebnis, eine Konvention, begründet durch eine Resolution des Unterhauses sei die beste Vorgehensweise. ${ }^{76}$ Nachdem sich 2008 auch das Joint Committee beider Häuser für eine detaillierte konventionsbegründende Resolution ${ }^{77}$ aussprach $^{78}$, erfolgte die offizielle Festlegung auf dieses Modell ${ }^{79}$ und einen konkreten Text. ${ }^{80}$

Danach soll der Zustimmungsvorbehalt für Einsätze außerhalb des Vereinigten Königreichs, die dem humanitären Völkerrecht unterliegen, gelten (section 1). Das Initiativrecht soll dem Premierminister obliegen, der seinem Antrag Informationen über Ziele, Orte und Rechtsfragen des Einsatzes nach eigenem Ermessen beifügen soll (section 2 sub-section 3). Diesem Antrag soll das Unterhaus durch Resolution zustimmen (s. 2 ss. 4), die Lords können zuvor angehört werden (s. 2 ss. 5). Nach s. 3 ss. 1 bis 5 gelten Ausnahmen für Eilfälle und Geheimhaltung, wenn sonst nach Ansicht des Regierungschefs Effektivität oder

69 Vgl. Select Committee on the Constitution, a.a.O. (Fn. 13), S. 17 ff.

70 Vgl. ebenda, S. $21 \mathrm{ff}$.

71 Vgl. ebenda, S. 42.

72 Reaktion abgedruckt in Select Committee on the Constitution, Waging War: Follow up, London 2007, S. 6 f. Die Antwort auf den sehr umfangreichen Bericht des SCC umfasst eineinhalb Seiten und wurde daher vom SCC indigniert als ,inadequate” gescholten.

73 Vgl. Secretary of State for Justice, The Governance of Britain, London 2007, S. 6.

74 Vgl. ebenda, S. 15.

75 Diese waren darüber „particularly pleased”, vgl. Select Committee on the Constitution, The Governance of Britain, London 2007, S. 3 f.

76 Vgl. Secretary of State for Justice, The Governance of Britain, a.a.O. (Fn. 73), S. 19.

$77 \mathrm{Ob}$ eine Konvention überhaupt durch Resolution begründet werden kann, ist zweifelhaft, vgl. dagegen David Jenkins, a.a.O. (Fn. 41), S. 259; wohl dafür O. Hood Phillips / Paul Jackson, a.a.O. (Fn. 18), S. 120.

78 Vgl. Joint Committee on the Draft Constitutional Reform Bill, Draft Constitutional Renewal Bill: Report, London 2008, S. 96.

79 Vgl. Secretary of State for Justice, The Governance of Britain - Constitutional Renewal, London 2008, S. 50.

80 Vgl. ebenda, S. 53 ff. 
Sicherheit gefährdet wäre. Nach der Einsatzentscheidung soll allerdings das Parlament informiert werden, wenn nicht nach Einschätzung des Premierministers Sicherheitsbelange dagegen sprechen (s. 3 ss. 8 bis 11). Der Einsatz von Spezialkräften ist nach dem Entwurf von jeder Zustimmung oder Information komplett ausgenommen (s. 4). Auch ist nach s. 5 keine Zustimmung erforderlich, wenn das Unterhaus zum betreffenden Zeitpunkt aufgelöst ist. Bei Eil- und Geheimhaltungsfällen wurde bewusst auf eine nachträgliche Befassung des Parlaments verzichtet. Grund dafür war die Angst vor negativen Auswirkungen auf die Truppe bei einer nachträglichen Versagung der Zustimmung. ${ }^{81}$

Wie die derzeitige konservativ-liberale Koalitionsregierung die Reform in dieser Frage weiterführen wird, ist ungewiss. Zwar wurden im Constitutional Reform and Governance Act 2010 viele Themen der Governance-of-Britain-Berichte umgesetzt ${ }^{82}$, nicht aber die Frage der Parlamentsbeteiligung, die bereits früher aus dem allgemeinen Prozess herausgelöst und wegen der Entscheidung gegen Gesetzgebung gesondert verfolgt wurde. ${ }^{83}$ Aktuelle Stellungnahmen zu dieser Frage von den regierenden Parteien liegen nicht vor; zwar wird in älteren Informationspapieren der Liberal Democrats eine Übertragung der Befugnisse auf das Parlament (und damit Gesetzgebung) gefordert ${ }^{84}$, aktuell taucht diese Forderung jedoch nicht mehr auf. ${ }^{85}$ Die Conservatives verhalten sich in ihrem Manifest lediglich generell zu Fragen der Prärogative. So ist „making the use of the royal Prerogative subject to greater democratic control so that Parliament is properly involved in all big national decisions" 86 als Ziel angegeben. Auch findet sich in der Koalitionsvereinbarung kein Hinweis auf eine weitere Reform dieses Gebiets. ${ }^{87}$ Dies ließe sich vorsichtig dahingehend interpretieren, dass auch unter der neuen Regierung keine gesetzliche Regelung, aber dennoch eine parlamentsstärkende Reform der Prärogative auf dem Gebiet des Streitkräfteeinsatzes beabsichtigt ist. Auch nimmt der Regierungswechsel den bisherigen Ansichten beider Häuser und ihrer Ausschüsse nicht jegliches Gewicht: Insbesondere die Lords sind in ihrer Zusammensetzung von Regierungswechseln nicht unmittelbar betroffen ${ }^{88}$, so dass ihre Position vom Regierungswechsel unbeeinflusst bleiben dürfte. Danach ist wohl von einer Weiterverfolgung der Möglichkeit auszugehen, durch Resolution eine Konvention zu etablieren. Entsprechend wird dem folgenden Vergleich der schon beschriebene Entwurf zugrunde gelegt.

81 Vgl. ebenda, S. 51.

82 Dazu gehören etwa der öffentliche Dienst und die Ratifikation völkerrechtlicher Verträge, http:// www.legislation.gov.uk/ukpga/2010/25/pdfs/ukpga_20100025_en.pdf (Abruf am 28. Mai 2011).

83 Vgl. Secretary of State for Justice, a.a.O. (Fn. 36), S. 13.

84 Vgl. Liberal Democrats, Policy Briefing: Constitutional Affairs, London 2009, http://www.libdems.org.uk/siteFiles/resources/PDF/Policy\%20Briefing\%20-\%20Constitutional\%20Affairs\%20 Oct\%2009.pdf (Abruf am 28. Mai 2011).

85 So enthält das aktuelle Papier zur politischen Reform keinen Bezug zu dieser Frage mehr, vgl. Liberal Democrats, Policy Briefing: Political Reform, http://www.libdems.org.uk/siteFiles/resources/PDF/Election\%20Policy/23\%20-\%20Political\%20Reform.pdf (Abruf am 28. Mai 2011).

86 David Cameron, Invitation to Join the Government of Britain, London 2010, http://media.conservatives.s3.amazonaws.com/manifesto/cpmanifesto2010_lowres.pdf (Abruf am 28. Mai 2011), S. 67.

87 Vgl. HM Government, The Coalition: Our Programme for Government, London 2010, http:// www.conservatives.com/ /media/Files/Downloadable\%20Files/coalition-programme.ashx?dl $=$ true (Abruf am 28. Mai 2011).

88 Vgl. Colin Turpin / Adam Tomkins, a.a.O. (Fn. 11), S. 642 ff. 


\section{Vergleich mit der Bundesrepublik Deutschland}

\subsection{Entstehung der Regelungen zum Streitkräfteeinsatz}

Der erste auffällige Unterschied in der rechtlichen Ausgestaltung der Parlamentsbeteiligung an Auslandseinsätzen von Streitkräften in Deutschland und dem Vereinigten Königreich liegt in der Entstehung der jeweiligen Regelungen.

Mit der Herleitung des Parlamentsvorbehalts aus der Verfassung hat sich das Bundesverfassungsgericht (BVerfG) zur Zielscheibe einiger methodischer Kritik gemacht. ${ }^{89}$ Die Ursache für den eher freien Umgang mit der juristischen Methode wurde im Nachgeben gegenüber politischem Druck ausgemacht. ${ }^{90}$ Neben der methodischen Kritik fand die Lösung des Bundesverfassungsgerichts in der Sache aber auch einigen Zuspruch. So biete das „salomonische" ${ }^{\text {91 }}$ Urteil mit dem Parlamentsvorbehalt einen Ausgleich für die „Öffnung” der Verfassung für Auslandseinsätze. ${ }^{92}$ Damit sei die „verfassungsrechtliche Entdeckung” 93 des Parlamentsvorbehalts ein entscheidender Beitrag zur Befriedungswirkung des Urteils. ${ }^{94}$

Die verfassungsgerichtliche Klärung dieser Frage kann man mit Rafael Biermann als „Verrechtlichung einer eigentlich politischen Kontroverse" 95 bezeichnen; dabei traf das Gericht eine Entscheidung, die nach dem System des Grundgesetzes dem verfassungsändern-

89 Siehe etwa Christian Burkiczak, Ein Entsendegesetz für die Bundeswehr?, in: Zeitschrift für Rechtspolitik, 36. Jg. (2003), H. 3, S. 82 - 86, S. 84; Volker Epping, a.a.O. (Fn. 1), S. 445 ff.; ders., Wehrverfassung. Entmilitarisierung, Wiederbewaffnung, Leistungsfähigkeit, in: Bodo Pieroth, Verfassungsrecht und soziale Wirklichkeit in Wechselwirkung, Berlin 2000, S. 183 208, S. 205; ders., in: ders. / Christian Hillgruber, Beck'scher Onlinekommentar zum Grundgesetz, Art. 87a Rn. 24 (Stand der Bearbeitung: 2010); Georg Nolte, Bundeswehreinsätze in kollektiven Sicherheitssystemen: Zum Urteil des Bundesverfassungsgerichts vom 12. Juli 1994, in: Zeitschrift für ausländisches öffentliches Recht und Völkerrecht, 54. Jg. (1994), S. 652 - 685, S. 683; Marcus Schultz, a.a.O. (Fn. 8), S. 436; Torsten Stein / Holger Kröninger, Bundeswehreinsatz im Rahmen von NATO-, WEU- bzw. VN-Militäraktionen. BVerfG vom 12. 7. 1994 2 BvE 3/93, 2 BvE 5/93, 2 BvE 7/93, 2 BvE 8/93, in: Jura, 17. Jg. (1995), H. 5, S. 254 - 262, S. 259; Mark Zimmer, a.a.O. (Fn. 8), S. 220 f.

90 Vgl. Christoph Gramm, Die Stärkung des Parlaments in der Wehrverfassung: Zu neueren Entscheidungen des Bundesverfassungsgerichts, in: Deutsches Verwaltungsblatt, 123. Jg. (2009), H. 23, S. $1476-1480$, S. 1478.

91 Vgl. Marcus Schultz, a.a.O. (Fn. 8), S. 432.

92 Vgl. Christian Burkizcak, AWACS II - In dubio pro Bundestag, in: Neue Zeitschrift für Verwaltungsrecht, 27. Jg. (2008), H. 7, S. 752 - 754, S. 753; Christoph Gramm, a.a.O. (Fn. 90), S. 1476; Torsten Stein / Holger Kröninger, a.a.O. (Fn. 89), S. 259 f.; Joachim Wieland, Die Entwicklung der Wehrverfassung, in: Neue Zeitschrift für Wehrrecht, 47. Jg. (2006), H. 3, S. 133 139, S. 137.

93 Christoph Gramm, Militärische Routine oder bewaffneter Einsatz? Anmerkungen zum Beschluss des Bundesverfassungsgerichts vom 25. März 2003: AWACS II, in: Unterrichtsblätter für die Bundeswehrverwaltung, 42. Jg. (2003), H. 5, S. 161 - 163, S. 163.

94 Vgl. Georg Nolte, a.a.O. (Fn. 89), S. 653, siehe auch Marcus Schultz, a.a.O. (Fn. 8), S. 436.

95 Rafael Biermann, a.a.O. (Fn. 7), S. 609. Volker Röben, Der Einsatz der Streitkräfte nach dem Grundgesetz, in: Zeitschrift für ausländisches öffentliches Recht und Völkerrecht, 63. Jg. (2003), S. 585 - 603, S. 585, sieht darin gar den Ausdruck einer „bundesrepublikanische[n] Tradition” der Verschiebung politischer Probleme nach Karlsruhe. Ähnlich Sven Gareis, Deutsche Außenpolitik: Grundlagen, Akteure, Strukturen und Prozesse, in: Informationen zur politischen Bildung 304/2009, S. 4 - 13, S. 12. 
den Gesetzgeber obliegt. ${ }^{96}$ Die Befassung des BVerfG mit einer Lösung eigentlich politischer Probleme birgt die Gefahr seiner Überforderung ${ }^{97}$ - das Gericht hat diese aber geschickt abgewendet und durch die Forderung nach einer gesetzlichen Ausgestaltung „den Ball ins politische Feld zurückgespielt" ${ }^{\prime 98}$. Eine so aktive Rolle kann sich das BVerfG aufgrund zweier Faktoren leisten, ohne den gesellschaftlichen Frieden zu gefährden: Einerseits ist es bereits durch das Grundgesetz selbst mit einer hervorgehobenen Stellung ausgestattet $^{99}$, andererseits genießt es wie kaum eine andere staatliche Institution das Vertrauen eines Großteils der Bevölkerung. ${ }^{100}$

Während in Deutschland die Frage des Streitkräfteeinsatzes durch das Bundesverfassungsgericht vom bestehenden Normensystem aus betrachtet und diesem unterworfen wurde, wird dieser Bereich im Vereinigten Königreich als weitgehend nicht reguliert angesehen. Hier wird ein grundlegender Unterschied in der juristischen Denkweise in beiden Staaten deutlich: Während das BVerfG ein System abstrakter Normen auf jeden denkbaren Sachverhalt anwendet, sind britische Richter eher bereit, rechtliche „black boxes” anzuerkennen, deren Inhalt nicht kontrollierbar ist. Daraus folgt, dass eine Regulierung in Großbritannien nicht durch Einordnung in ein vorhandenes System erreicht werden kann, sondern nur im Rahmen des politischen Prozesses: „complaints must be aired at [...] the ballot box"101. Am Ende dieses Prozesses wird wie oben beschrieben aller Wahrscheinlichkeit nach eine Resolution des Unterhauses stehen, die eine entsprechende Konvention begründen soll.

\subsection{Geltung und prozessuale Durchsetzbarkeit}

Ein weiterer wesentlicher Unterschied zwischen der Regelung im Vereinigten Königreich und der in Deutschland liegt in der Geltung, die diese beansprucht und in der daraus resultierenden prozessualen Durchsetzbarkeit. Während in Deutschland eine informelle parla-

96 Vgl. Manfred Baldus, Braucht Deutschland eine neue Wehrverfassung?, in: Neue Zeitschrift für Wehrrecht, 48. Jg. (2007), H. 4, S. 133 - 138, S. 134 ff.; Christian Burkiczak, a.a.O. (Fn. 89), S. 86; Christoph Gramm, a.a.O. (Fn. 90), S. 1480; Markus Krajewski, Das „Parlamentsheer“ als Kollateralschaden des Irak-Kriegs? Zum AWACS-Beschluss des Bundesverfassungsgerichts vom 25.3.2003, 2 BvQ 18/03, in: Archiv des Völkerrechts, 41. Jg. (2003), H. 4, S. 419 - 425, S. 425; Joachim Wieland, a.a.O. (Fn. 92), S. 138 f.; Karl Ullrich Voss, Rechtsstaat ad hoc? Anwendung von Gesetzesvorbehalt und Parlamentsvorbehalt bei Auslandseinsätzen der Bundeswehr, in: Zeitschrift für Rechtspolitik, 40. Jg. (2007), H. 3, S. 78 - 81, S. 81; vorsichtiger Torsten Stein / Holger Kröninger, a.a.O. (Fn. 89), S. 259, S. 262.

97 Vgl. Georg Nolte, a.a.O. (Fn. 89), S. 653.

98 Sven Gareis, a.a.O. (Fn. 95), S. 12.

99 Insbesondere ist es zur Schlichtung von Streitigkeiten zwischen Verfassungsorganen (und damit zumindest auch politischen Streitigkeiten) nach Art. 93 Abs. 1 Nr. 1 bis 4 und Abs. 2 GG berufen, und seine Urteile können Gesetzeskraft haben, Art. 94 Abs. 2 S. 1 i.V.m. $\$ 31$ BVerfGG.

100 So führt es zusammen mit Polizei und Bundeswehr die Liste der Einrichtungen, denen die Bundesbürger am meisten vertrauen, an: 88 Prozent der Befragten einer Studie gaben an, dem Bundesverfassungsgericht zumindest "eher” zu vertrauen, und 90 Prozent bescheinigten ihm eine gute Aufgabenerfüllung. Zum Vergleich: dem Bundestag vertrauten 58 Prozent, 55 Prozent bescheinigten ihm eine gute Aufgabenerfüllung; vgl. Rüdiger Fiebig / Carsten Pietsch, Die Deutschen und ihre Streitkräfte, in: APuZ B 48/2009, S. 36 - 41, S. 38 f.

101 Sir Anthony Clarke, a.a.O. (Fn. 59), S. 715. 
mentarische Übung für unzureichend befunden wurde ${ }^{102}$, wäre die vorgeschlagene Unterhaus-Resolution nicht gerichtlich durchsetzbar, sondern aus rechtlicher Sicht lediglich eine „non-binding plea" 103 mit „(not actually legal) force" 104 .

Jedoch können auch ungeschriebene oder nicht durchsetzbare Regeln die Rechtskultur entscheidend beeinflussen ${ }^{105}$; daher ist die Einbeziehung gesellschaftlicher Hintergründe bei der Rechtsvergleichung von besonderer Bedeutung. ${ }^{106}$ Wenn Konventionen durch ihre politische Bindung ähnlich starke faktische Geltung hätten wie das deutsche Verfassungsrecht wäre das „law in action“ trotz theoretisch sehr unterschiedlicher Normen ähnlich. Bezüglich der Konventionen ist dies jedoch nicht der Fall. In der Vergangenheit wurden diese bereits vielfach, insbesondere durch die Regierung unter Margaret Thatcher, gebrochen. ${ }^{107}$ Insofern unterscheidet sich die tatsächliche Geltung der vorgeschlagenen Resolution nicht wesentlich von der rechtlichen Geltung.

Im Gegensatz dazu ist das Vorliegen der Voraussetzungen des Parlamentsvorbehalts in Deutschland „gerichtlich voll überprüfbar"108. Damit ist die Einbeziehung des Parlaments in diesem Bereich in Deutschland deutlich stärker abgesichert als im Vereinigten Königreich.

\subsection{Reichweite}

Neben der Entstehung und Durchsetzbarkeit der Beteiligungsregelungen ist vor allem deren Reichweite von entscheidender Bedeutung. Hier zeigt sich das konkrete Ergebnis der jeweils getroffenen Abwägung zwischen Effektivität des Einsatzes und demokratischer Kontrolle.

(a) Einsatzbegriff

$\mathrm{Zu}$ definieren, was eine Beteiligung ${ }^{109}$ auslöst, ist besonders schwierig, weil Einsätze im Detail schwer vorhersehbar sein können - daher wird große Flexibilität gefordert. ${ }^{110}$ Prob-

102 So durch die Fraktion der FDP, BT-Drs. 15/1985, S. 4, aber auch durch die von SPD und Bündnis 90 / Die Grünen, BT-Drs. 15/2742, S. 1.

103 David Jenkins, a.a.O. (Fn. 2), S. 157.

104 Ders., a.a.O. (Fn. 41), S. 259.

105 Vgl. Edward Eberle, The Method and Role of Comparative Law, in: Washington University Global Studies Law Review, 8. Jg. (2009), H. 3, S. 451 - 486, S. 474.

106 Vgl. ebenda, S. 452.

107 Vgl. Iain Byrne / Stuart Weir, a.a.O. (Fn. 14), S. 453.

108 BVerfGE 121, S. 135 (S. 168).

$109 \mathrm{Vgl}$. David Jenkins, a.a.O. (Fn. 2), S. 149; Dieter Wiefelspütz, Das Parlamentsbeteiligungsgesetz vom 18. 3. 2005, in: Neue Zeitschrift für Verwaltungsrecht, 24. Jg. (2005), H. 5, S. $496-500$, S. 496.

110 Vgl. Peter Dreist, Ein Gesetz gewinnt Konturen: zum notwendigen Regelungsgehalt eines künftigen Parlamentsbeteiligungsgesetzes, in: Zeitschrift für Gesetzgebung, 19. Jg. (2004), H. 1, S. 39 - 62, S. 54; Martin Nolte, Der AWACS-Einsatz in der Türkei zwischen Parlamentsvorbehalt und Regierungsverantwortung, in: Neue Juristische Wochenschrift, 56. Jg. (2003), H. 33, S. 2356 - 2360, S. 2359; Michael Wild, Verfassungsrechtliche Möglichkeiten und Grenzen für Auslandseinsätze der Bundeswehr nach dem Kosovo-Krieg - Versuch einer Systematisierung, in: 
lematisch ist insbesondere die Einbeziehung von militärischen Handlungsformen, die unterhalb des „Krieges” im umgangssprachlichen Sinn angesiedelt sind. ${ }^{111}$

Während das Grundsatzurteil des BVerfG lediglich vom „Einsatz bewaffneter Streitkräfte" spricht ${ }^{112}$ und dies nicht weiter eingegrenzt hat ${ }^{113}$, gibt es über den Begriff des Einsatzes im deutschen Schrifttum eine umfassende Diskussion. Dabei lassen sich zwei Kriterien bezüglich der Tätigkeit der Soldaten herausarbeiten: die Anwendung von Zwang und die Nutzung „spezifischer” oder "typischer” militärischer Strukturen oder Fähigkeiten. Diese werden teils einzeln ${ }^{114}$, teils kumulativ ${ }^{115}$ und teils alternativ herangezogen. ${ }^{116}$ Auch werden verbreitet nur Einsätze als umfasst angesehen, die „ihrer Prägung nach auf eine Auseinandersetzung mit kriegs- oder doch kriegsähnlichem Charakter zielen"117.

Die gesetzliche Regelung konkretisiert den Begriff des Einsatzes hinsichtlich einiger be-

Die Öffentliche Verwaltung, 53. Jg. (2000), H. 15, S. 622 - 631, S. 631; Christoph Gramm, a.a.O. (Fn. 93), S. 163, spricht sich deshalb für eine weite Definition aus, die aber im Zweifel für die Parlamentsbeteiligung auszulegen sei.

111 Vgl. Peter Dreist, AWACS-Einsatz ohne Parlamentsbeschluss? Aktuelle Fragestellungen zur Zulässigkeit von Einsätzen bewaffneter Streitkräfte unter besonderer Berücksichtigung der NATOAWACS-Einsätze in den USA 2001 und in der Türkei 2003, in: Zeitschrift für ausländisches öffentliches Recht und Völkerrecht, 64. Jg. (2004), S. 1001 - 1043, S. 1035.

112 Vgl. BVerfGE 90, S. 286 (S. 381).

113 Vgl. Peter Dreist, Offene Rechtsfragen des Einsatzes bewaffneter deutscher Streitkräfte - Zwischenbilanz und Problemaufriss, in: Neue Zeitschrift für Wehrrecht, 43. Jg. (2002), H. 4, S. 133 - 154, S. 144; ders., Prüfschema: Einsatz bewaffneter deutscher Streitkräfte im Ausland, in: Neue Zeitschrift für Wehrrecht, 44. Jg. (2003), H. 4, S. 153 - 166, S. 155 f.

114 Für Zwang als maßgebliches Kriterium Claus Arndt, Bundeswehr und Polizei im Notstand, in: Deutsches Verwaltungsblatt, 83. Jg. (1968), H. 18, S. 729 - 732, S. 729; Stefan Oeter, Einsatzarten der Streitkräfte außer zur Verteidigung - Verfassungsrechtliche Grundlagen, in: Neue Zeitschrift für Wehrrecht, 41. Jg. (2000), H. 2, S. 89 - 101, S. 96; Marcus Schultz, a.a.O. (Fn. 8), S. 178; Wolfgang Weiß, a.a.O. (Fn. 2), S. 104; Michael Wild, a.a.O. (Fn. 110), S. 624; für die Nutzung militärischer Fähigkeiten Ferdinand Kirchhof, in: Josef Isensee / ders., Handbuch des Staatsrechts der Bundesrepublik Deutschland, Heidelberg 1996, S. 994.

115 Vgl. Otto Depenheuer, in: Theodor Maunz / Günter Dürig (Hrsg.), Grundgesetz. Kommentar, Art. 87a Rn. 169 (Stand der Bearbeitung: 2008); Christian Fischer / Andreas Fischer-Lescano, Enduring Freedom für Entsendebeschlüsse? Völker- und verfassungsrechtliche Probleme der deutschen Beteiligung an Maßnahmen gegen den Internationalen Terrorismus, in: Kritische Vierteljahresschrift für Gesetzgebung und Rechtswissenschaft, 85. Jg. (2002), H. 1, S. 113 144, S. $117 \mathrm{f}$.

116 Vgl. Volker Epping, in: ders. / Christian Hillgruber, a.a.O. (Fn. 89), Art. 87a Rn. 17; Florian Schröder, Das parlamentarische Zustimmungsverfahren zum Auslandseinsatz der Bundeswehr in der Praxis, Köln 2005, S. 203.

117 Dieter Wiefelspütz, Der Einsatz der Streitkräfte und die konstitutive Beteiligung des Deutschen Bundestags, in: Neue Zeitschrift für Wehrrecht, 44. Jg. (2003), H. 3, S. 133 - 151, S. 140; ders., Bewaffnete Einsätze der Bundeswehr auf See, in: Neue Zeitschrift für Wehrrecht, 46. Jg. (2005), H. 4, S. 146 - 163, S. 163; ders., Das Parlamentsheer, Berlin 2005, S. 423. Entsprechend Otto Depenheuer, in: Theodor Maunz / Günter Dürig (Hrsg.): Grundgesetz. Kommentar, Art. 87a Rn. 171 (Stand der Bearbeitung: 2008) und für „einiges Gewicht” der zustimmungsbedürftigen Einsätze Peter Dreist, Der Bundestag zwischen „Vorratsbeschluss” und Rückholrecht: Plädoyer für ein wirkungsvolles Parlamentsbeteiligungsgesetz, in: Kritische Vierteljahresschrift für Rechtswissenschaft, 87. Jg. (2004), H. 1, S. 79 - 119, S. 91; Roman Schmidt-Radefeldt, Parlamentarische Kontrolle der internationalen Streitkräfteintegration, Berlin 2005, S. 160 f.; gegen die Ausnahme lediglich polizeilicher „Einsätze” Volker Epping, in: ders. / Christian Hillgruber, a.a.O. (Fn. 89), Art. 87a Rn. 29.3. 
stimmter Handlungsformen: Nach $\$ 2$ Abs. 2 ParlBG sind „vorbereitende Maßnahmen und Planungen” sowie „humanitäre Hilfsdienste und Hilfsleistungen der Streitkräfte”, solange sie lediglich zur Verteidigung bewaffnet sind, vom Einsatzbegriff ausgenommen. Im Rückschluss aus $\$ 4$ Abs. 3 ParlBG ergibt sich aber, dass „vorbereitende Maßnahmen” keine Erkundungskommandos beinhalten ${ }^{118}$ (diese sind aber dem vereinfachten Verfahren unterworfen). Daneben sind auch einzelne Soldaten in Austauschprogrammen und Stäben internationaler Organisationen nach $\$ 4$ Abs. 3 ParlBG grundsätzlich „Einsätze”. Damit hat sich der Gesetzgeber gegen eine enge, am (umgangssprachlichen) Kriegsbegriff orientierte Definition und für die Einbeziehung der Verwendung auch einzelner Soldaten im Ausland entschieden.

Einen anderen Weg geht die Definition im Vereinigten Königreich. Nach s. 1 ss. 2 der Draft Detailed War Powers Resolution ist die Parlamentsbeteiligung für den Einsatz von Gewalt („force“) durch britische Truppen außerhalb des Vereinigten Königreichs nur erforderlich, wenn die entsprechende Situation dem humanitären Völkerrecht unterworfen ist. Damit ist allerdings das Definitionsproblem auf den Begriff des ,armed conflict“ im humanitären Völkerrecht verlagert. ${ }^{119}$ Diesen definierte der Internationale Strafgerichtshof für das ehemalige Jugoslawien 1995 als „whenever there is a resort to armed force between states or protracted armed violence between governmental authorities and organized armed groups or between such groups within a state" ${ }^{120}$. Folgt man dieser Definition, ist ein bestimmter Organisations- und Ausrüstungsgrad aufseiten der potenziellen Gegner erforderlich. Ein bewaffneter Einsatz, der sich aber nicht gegen organisierte bewaffnete Gruppen richtet, wäre demnach ausgenommen. Ebenfalls erfasst der Regierungsentwurf keine militärischen Handlungsformen, die lediglich die Gefahr einer Beteiligung an bewaffneten Konflikten etwa durch Verschlechterung der Lage im Einsatzgebiet („mission creep“) bergen. ${ }^{121}$ Demgegenüber hatte ein Vorschlag von 2007 noch die „real likelihood of [...] activities leading to the participation of armed forces in armed conflict"122 ausreichen lassen. Auch fällt das „Peacekeeping“, also die Überwachung des Friedens nach einem bewaffneten Konflikt, nicht in die Definition der Resolution ${ }^{123}$, ebenso wenig Austauschprogramme, ständige Stäbe in internationalen Organisationen, Hilfsmaßnahmen und vorbereitende Maßnahmen, wenn noch kein bewaffneter Konflikt besteht.

Zwar haben die britischen und deutschen Regelungen gemeinsam, dass die Abgrenzung eines Auslöserkriteriums für die Beteiligung des Parlaments im Einzelnen unklar ist, jedoch ist die britische Definition durch die Anknüpfung an den völkerrechtlichen Begriff des bewaffneten Konflikts jedenfalls deutlich enger und nimmt weite Bereiche (die „Einsatz” im deutschen Sinn, aber nicht bewaffneter Konflikt sind) aus. Entsprechend ist hier auch die Stellung des deutschen Parlaments gegenüber der Regierung deutlich stärker.

118 Vgl. Wolfgang Weiß, a.a.O. (Fn. 2), S. 105.

$119 \mathrm{Zu}$ daraus entstehenden Problemen siehe Secretary of State for Justice, The Governance of Britain: War Powers and Treaties, Limiting Executive Powers, London 2007, S. 25.

120 Decision on the Defence Motion for Interlocutory Appeal on Jurisdiction, Prosecutor v. Tadic, Case No. IT-94- 1, A.C., 2. Oktober 1995, Absatz 70.

121 Vgl. Claire Taylor / Richard Kelly, a.a.O. (Fn. 43), S. 33.

122 Secretary of State for Justice, a.a.O. (Fn. 119), S. 44.

$123 \mathrm{Vgl}$. Claire Taylor, Armed Forces (Parliamentary Approval for Participation in Armed conflict) Bill, Research Paper 05/56, London 2005, http://www.parliament.uk/briefing-papers/RP05-56. pdf (Abruf am 29. Mai 2011), S. $14 \mathrm{f}$. 
(b) Zeitpunkt der Beteiligung

Auch bezüglich des Zeitpunkts, zu dem das Parlament an der Entscheidung beteiligt wird, bestehen Unterschiede zwischen der deutschen und der britischen Regelung. Zwar sehen beide vor, dass das Parlament grundsätzlich vor einem Einsatz beteiligt werden soll ( $\$ \$ 1$ Abs. 2, 3 Abs. 1 ParlBG und s. 1 ss. 1 der Draft Resolution), jedoch unterscheiden sie sich signifikant bezüglich der Beteiligung des Parlaments nach einer Eilentscheidung. Bei Ausnahmen vom Grundsatz der vorherigen Befassung („emergency and security conditions“) sieht die britische Regelung so keine mitbestimmende Beteiligung mehr vor. Grund dafür ist nach der Begründung des Entwurfs, dass eine Versagung oder Missbilligung ex post „very serious and undesirable consequences” haben und die Glaubwürdigkeit, die internationalen Beziehungen des Vereinigten Königreichs sowie die Sicherheit und Moral der Truppen beeinträchtigen könnte. ${ }^{124}$ In diesen Fällen bleiben lediglich die Informationsrechte beim Unterhaus. Diese Beeinträchtigungen zu tragen ist man in Deutschland bereit: Auch bei Eilfällen ist der Bundestag nach Beginn des Einsatzes zu befassen und kann diesen beenden $^{125}$; zudem erlaubt das Rückholrecht nach $\$ 8$ ParlBG dem Bundestag, „Herr seiner Zustimmungsentscheidung” zu bleiben. ${ }^{126}$ Ein entsprechendes Rückholrecht gibt es entgegen früherer Entwürfe ${ }^{127}$ in der britischen Draft Resolution jedoch nicht; aus oben genannten Gründen ist eine Beteiligung nach Beginn einer Operation ausdrücklich nicht vorgesehen. ${ }^{128}$ Damit ist das Zeitfenster für eine Beteiligung im Vereinigten Königreich sehr beschränkt, während der Bundestag nicht nur auch bei Eilfällen beteiligt werden muss, sondern sogar jederzeit im Nachhinein in der Lage ist, einen Einsatz zu beenden.

\section{(c) Entscheidung in Eilfällen}

Bezüglich der Entscheidung in Eilfällen weisen die Regelungen gewisse Parallelen auf. Nach $\$ 5$ Abs. 1 ParlBG und s. 3 ss. 1 Draft Resolution kann die Regierung diese ohne eine Beteiligung des Parlaments beschließen. Dies war in Deutschland bereits im Grundsatzurteil des Bundesverfassungsgerichts angelegt ${ }^{129}$ und damit für den deutschen Gesetzgeber auch nicht disponibel. In beiden Staaten soll die Regierung in diesem Fall das Parlament informieren ( $\$ 5$ Abs. 2 ParlBG und s. 3 ss. 9 Draft Resolution). Wesentlicher Unterschied ist allerdings, dass sich in Deutschland eine nachträgliche Befassung anschließt $(\$ 5$ Abs. $3 \mathrm{~S}$. 1 ParlBG) ${ }^{130}$ und dass die Feststellung eines Eilfalles im Vereinigten Königreich nach s. 3 ss. 5 Draft Resolution allein dem Premierminister obliegt. So ist auch hier trotz der gemeinsamen Ermächtigung, Eilfälle ohne eine vorherige Befassung des Parlaments durchzuführen, die britische Regelung wesentlich exekutiv-freundlicher.

124 Vgl. Secretary of State for Justice, a.a.O. (Fn. 79), S. 51.

125 Vgl. $\$ 5$ Abs. 3 ParlBG nach BVerfGE 90, S. 286 (S. 388).

126 Vgl. BVerfGE 124, S. 267 (S. 278).

127 Vgl. Andrew Blick / Paul Hunt / Stuart Weir, in: Select Committee on the Constitution, a.a.O. (Fn. 24), S. 90; Rodney Brazier, a.a.O. (Fn. 43), S. 35 f.

128 Vgl. Secretary of State for Justice, a.a.O. (Fn. 79), S. 51.

129 Vgl. BVerfGE 90, S. 286 (S. 388).

130 Dies ist auch nach dem Urteil des Bundesverfassungsgerichts „in jedem Fall”, damit selbst bei „Erledigung” des Einsatzes durchzuführen. Christian Lutze, Der Parlamentsvorbehalt beim Einsatz bewaffneter Streitkräfte, in: Die Öffentliche Verwaltung, 56. Jg. (2003), H. 23, S. 972 980, S. 978 verweist insofern auf die „vertrauensbegründende Komponente” des Bundestagsbeschlusses sowie auf die Wirkung für zukünftige Entscheidungen. 
(d) Geheimschutz und Informationsrecht

Ein weiteres Problemfeld ist das Spannungsverhältnis zwischen der Geheimhaltung bestimmter Informationen und dem Erfordernis einer möglichst umfassenden Information des Parlaments. Die Anträge der Regierungen sind sehr allgemein gefasst ${ }^{131}$ und enthalten zum Beispiel keine Informationen über die Umstände im Einsatzgebiet oder die verfolgte Strategie. Diesbezüglich besteht ein Wissensvorsprung der Regierung ${ }^{132}$, ausreichende Information ist aber eine wesentliche Voraussetzung für die Ausübung des Parlamentsvorbehalts. ${ }^{133}$ Dem steht oftmals eine Praxis gegenüber, die etwa ein Vorbereiten von Zeugenaussagen oder die Zurückhaltung von Informationen beinhaltet. ${ }^{134}$ Andernfalls muss die Regierung mit Vergrößerung des eingeweihten Personenkreises die Weitergabe von geheimen Informationen fürchten. ${ }^{135}$

Dem Bedürfnis nach Information des Parlaments kommt das ParlBG nach, indem es gemäß $\$ 3$ Abs. 2 relativ detaillierte Anforderungen an den Antrag der Regierung stellt und durch $\$ 6$ eine „regelmäßige” Unterrichtungspflicht über „den Verlauf des Einsatzes und die Entwicklungen im Einsatzgebiet” begründet. Im Vereinigten Königreich sind dagegen sämtliche Informationen im Antrag und im Bericht bei Ausnahmefällen lediglich nach Ermessen des Premierministers gestaltet. ${ }^{136}$ Damit kann dieser nicht nur faktisch (durch seinen Wissensvorsprung), sondern auch rechtlich den Informationsfluss kontrollieren. ${ }^{137}$ Eine dauernde Informationspflicht über laufende Einsätze besteht nicht, ist aber auch im System der Draft Resolution nicht erforderlich, da das Parlament auf diese Einsätze keinerlei Einfluss hat.

In beiden Systemen sind die Abgeordneten für die Erfüllung ihres Kontrollauftrags auf die Kooperationsbereitschaft der Kontrollierten angewiesen: eine Prüfung der von der Regierung zugänglich gemachten Informationen ist dem einzelnen Abgeordneten kaum möglich. Die deutsche Rechtslage ist hier zwar parlamentsfreundlicher, indem sie eine Informationspflicht ohne Ermessen vorsieht - jedoch dürfte sich diese Pflicht angesichts der ebenfalls eingeschränkten Erkenntnismöglichkeiten der Rechtsprechung nur unzureichend gerichtlich durchsetzen lassen, sodass die Abgeordneten in beiden Ländern faktisch vor den gleichen Herausforderungen stehen.

131 Vgl. Dieter Blumenwitz, Einsatzmöglichkeiten der Bundeswehr im Kampf gegen den Terrorismus, in: Zeitschrift für Rechtspolitik, 35. Jg. (2002), H. 3, S. $102-106$, S. 106; vgl. auch $\$ 3$ Abs. 2 ParlBG; s. 2 ss. 3 Draft Resolution.

132 Vgl. BVerfGE 121, S. 135 (S. 159); Rafael Biermann, a.a.O. (Fn. 7), S. 611.

133 Vgl. Roman Schmidt-Radefeldt, a.a.O. (Fn. 117), S. 171.

134 Sondervotum der Fraktion Bündnis 90/Die Grünen in der Beschlussempfehlung und Bericht des Verteidigungsausschusses als 1. Untersuchungsausschuss gemäß Artikel 45a Abs. 2 GG, BTDrs. 16/10650, S. 169, S. 179; bezüglich des Vereinigten Königreichs Andrew Blick / Iain Byrne / Stuart Weir, a.a.O. (Fn. 44), S. 412.

135 So geschehen etwa bezüglich persönlicher Daten von KSK-Angehörigen, vgl. Beschlussempfehlung und Bericht des Verteidigungsausschusses, a.a.O. (Fn. 134), S. 135.

136 S. 2 ss. 3b bezüglich der Informationen bei Antragsstellung, s. 3 ss. 5 für das Vorliegen der „emergency“ oder „security condition“, s. 3 ss. 6 und 8 für die Beteiligung der Ausschüsse, s. 3 ss. $9 \mathrm{~b}$ bezüglich des Berichts bei Einsatz ohne Zustimmung, s. 3 ss. 11 bezüglich der Zurückhaltung des Berichts.

137 Vgl. David Jenkins, a.a.O. (Fn. 2), S. 157. 
Verbunden mit dem Informationsrecht ist die Frage, wie mit geheimhaltungsbedürftigen Einsätzen umzugehen ist. Bei bestimmten Einsätzen, bei denen es etwa maßgeblich auf das Überraschungsmoment ankommt, ist eine Plenarberatung „der Natur der Sache nach” ungeeignet ${ }^{138}$ : Der Gegner würde gewarnt und das Einsatzziel damit gefährdet. Im Vereinigten Königreich sieht s. 3 ss. 3 und 4 der Draft Resolution dafür eine weitreichende ${ }^{139}$ Ausnahme vom Prozess im Regelfall vor ${ }^{140}$ : Wenn eine Veröffentlichung von Informationen bezüglich der Entscheidung nach Ansicht des Premierministers die Effektivität der Streitkräfte oder die Sicherheit britischer oder verbündeter Streitkräfte oder Personen negativ beeinflussen („prejudice“) würde, ist keine Beteiligung des Parlaments erforderlich. Während diese Bedingung nach Ansicht des Regierungschefs anhält, ist auch keinerlei Information des Parlaments erforderlich (s. 3 ss. 11).

In Deutschland sieht das ParlBG keine ausdrückliche Regelung dieses Problems vor. ${ }^{141}$ Die Diskussion dreht sich daher um die Frage, ob das Bedürfnis der Geheimhaltung von der Ausnahme der "Gefahr im Verzug” erfasst ist. So wird verbreitet vertreten, auch eine Gefahr für den Zweck des Einsatzes durch Offenlegung von Informationen sei „Gefahr im Verzug" im Sinne der vom BVerfG begründeten ${ }^{142}$ und in $\$ 5$ Abs. 1 ParlBG aufgenommenen Ausnahme. ${ }^{143} \mathrm{Da}$ aber die Offenlegung von Informationen immer das Überraschungsmoment aufheben und so die Zweckerreichung potentiell beeinträchtigen kann, würde die Einbeziehung der Geheimhaltung in die „Gefahr im Verzug” zur Umkehrung der Ausnahme in die Regel führen. ${ }^{144}$ Daher spricht viel dafür, dass Gefahr im Verzug und Geheimhal-

138 Vgl. Gesetzentwurf der Fraktion der FDP vom 8. November 2006, BT-Drs. 16/3342, S. 4; ähnlich Peter Dreist, a.a.O. (Fn. 113), S. 146.

$139 \mathrm{Vgl}$. David Jenkins, a.a.O. (Fn. 2), S. 158.

140 Der Vorschlag etwa des New Politics Network, in: Select Committee on the Constitution, a.a.O. (Fn. 24), S. 95, S. 102 f., diese Fälle durch einen Ausschuss entscheiden zu lassen, hat sich demgegenüber nicht durchgesetzt.

141 Auch hier hat sich das Modell eines mit diesen Fällen befassten Ausschusses nicht durchgesetzt, dafür aber der Gesetzentwurf der Fraktion der FDP, a.a.O. (Fn. 138), S. 1 ff.; Minderheitenbericht der Fraktion der FDP in der Beschlussempfehlung und Bericht des Verteidigungsausschusses, a.a.O. (Fn. 134), S. 142; Georg Axer, Das „Kommando Spezialkräfte” zwischen Geheimschutzinteresse und Parlamentsvorbehalt, in: Zeitschrift für Rechtspolitik, 40. Jg. (2007), H. 3, S. 82 - 84, S. 83; Rafael Biermann, a.a.O. (Fn. 7), S. 626; Peter Dreist, a.a.O. (Fn. 113), S. 151; Timo Noetzel / Benjamin Schreer, Spezialkräfte der Bundeswehr: Strukturerfordernisse für den Auslandseinsatz, SWP-Studie, Berlin 2007, http://www.swp-berlin.org/fileadmin/contents/products/studien/2007_S26_ntz_srr_ks.pdf (Abruf am 29. Mai 2011), S. 18 f; Ronald Pofalla, Die Bundeswehr im Ausland - Eine Zwischenbilanz des Gesetzgebungsverfahrens, in: Zeitschrift für Rechtspolitik, 37. Jg. (2004), H. 7, S. 221 - 225, S. 225; Phillip Scherrer, a.a.O. (Fn. 7), S. 286. Eine solche Delegation wäre auch verfassungsrechtlich möglich, siehe Dieter Wiefelspütz, Die militärische Integration der Bundeswehr und der konstitutive Parlamentsvorbehalt, in: Zeitschrift für ausländisches öffentliches Recht und Völkerrecht, 64. Jg. (2004), S. 363 - 389, S. 385.

142 Vgl. BVerfGE 90, S. 286 (S. 388).

143 Vgl. Christian Burkiczak, a.a.O. (Fn. 89), S. 86; Volker Epping, a.a.O. (Fn. 1), S. 456; ders., a.a.O. (Fn. 89), S. 206; Christian Lutze, a.a.O. (Fn. 130), S. 977; Roman Schmidt-Radefeldt, a.a.O. (Fn. 117), S. 162 f.; Dieter Wiefelspütz, a.a.O. (Fn. 109), S. 499; ders., Das Parlamentsheer, a.a.O. (Fn. 117), S. 474.

144 Vgl. Volker Epping, a.a.O. (Fn. 1), S. 456; ders., a.a.O. (Fn. 89), S. 206; Phillip Scherrer, Das Parlament und sein Heer: Das Parlamentsbeteiligungsgesetz, a.a.O. (Fn. 7), S. 285; Roman Schmidt-Radefeldt, a.a.O. (Fn. 117), S. 166 f. 
tungsbedürfnis zwar breite Überschneidungen aufweisen ${ }^{145}$ - ein Einsatz bei Gefahr im Verzug wird regelmäßig geheim zu halten sein -, aber nicht deckungsgleich sind. So hat auch das BVerfG die Gefahr im Verzug nicht als Beispiel für Ausnahmen vom Grundsatz der vorherigen Befassung des Parlaments genannt, sondern als einzige Ausnahme ${ }^{146}$ : „Eine Einschätzungsprärogative kann der Exekutive lediglich für den Eilfall [...] überlassen sein." ${ }^{147}$ Auch ist die Gefahr im Verzug bereits als Ausnahme vom Grundsatz eng auszulegen. ${ }^{148}$ Dies spricht für eine Anknüpfung an den polizeirechtlichen Begriff, wonach die Eilbedürftigkeit maßgebliches Element ist. ${ }^{149}$ Damit ist in Deutschland keine Ausnahme für die Geheimhaltung vorgesehen. Somit ist auch hier die deutsche Regelung parlamentsfreundlicher als die britische Draft Resolution.

Ein Sonderproblem im Kontext der geheimhaltungsbedürftigen Einsätze stellt der Einsatz von Spezialkräften dar. Dessen Wichtigkeit wächst infolge der Zunahme asymmetrischer Konflikte. ${ }^{150}$ So nahmen etwa auch Teile des deutschen Kommandos Spezialkräfte an „direct action“151 im Rahmen der Operation Enduring Freedom in Südafghanistan teil ${ }^{152}$, was jedenfalls leicht als Widerspruch zu einer zweideutigen Zusicherung des Kanzlers verstanden werden kann, man werde sich nicht an den Kampfhandlungen beteiligen. ${ }^{153}$ Daneben gibt es Spekulationen über einen umfassenden Einsatz von deutschen Spezialkräften im Rahmen von ISAF ${ }^{154}$, wobei der Umfang der Beteiligung auch dem Parlament nicht bekannt ist. ${ }^{155}$ Die deutsche Praxis besteht derzeit darin, dass die Obleute, Ausschuss- und Fraktionsvorsitzenden freiwillig und vertraulich informiert werden. ${ }^{156}$ Eine entsprechende

145 Vgl. Peter Dreist, a.a.O. (Fn. 113), S. 144

146 Anders aber Willibald Hermsdörfer, Einsatz bewaffneter Streitkräfte vor Zustimmung des Deutschen Bundestags, in: Unterrichtsblätter für die Bundeswehrverwaltung, 42. Jg. (2003), H. 11, S. $404-406$, S. 404.

147 BVerfGE 121, S. 135 (S. 163). Vgl. auch BVerfGE 90, S. 286 (S. 388); BVerfGE 121, S. 135 (S. 154, S. 169).

148 Vgl. Andreas Gilch, Das Parlamentsbeteiligungsgesetz: Die Auslandsentsendung der Bundeswehr und deren verfahrensrechtliche Ausgestaltung, Nürnberg 2005, S. 125.

149Vgl. Ronald Pofalla, a.a.O. (Fn. 141), S. 223.

150 Vgl. Timo Noetzel / Benjamin Schreer, a.a.O. (Fn. 141), S. 7 f.; Susanne Gaschke, Unter Kriegern: Sind Elitesoldaten auf geheimer Mission die Zukunft der Bundeswehr? Ein Besuch beim Kommando Spezialkräfte, in: Die Zeit vom 29. Juli 2010, S. 3.

151 „Direct action“ ist Teil des Fähigkeitsspektrums von Spezialkräften und beinhaltet „offensive, zielgerichtete, räumlich und zeitlich eng umschriebene Einsätze, um Personen festzusetzen/zu befreien, Material/Einrichtungen in Besitz zu nehmen oder zu zerstören/schädigen”. Siehe Timo Noetzel / Benjamin Schreer, a.a.O. (Fn. 141), S. 9.

152 Vgl. Beschlussempfehlung und Bericht des Verteidigungsausschusses, a.a.O. (Fn. 134), S. 104, S. 111; Timo Noetzel / Benjamin Schreer, a.a.O. (Fn. 141), S. 15, S. 17; Grundlage dafür war die Zustimmung des Bundestags zum Antrag der Bundesregierung vom 7. November 2001, BTDrs. 14/7296, der von 3.900 eingesetzten Soldaten (größtenteils Seestreitkräfte) auch 100 Spezialkräfte vorsieht, um „Führungs- und Ausbildungseinrichtungen von Terroristen auszuschalten, Terroristen zu bekämpfen, gefangen zu nehmen und vor Gericht zu stellen”, S. 3 f.

153 „Es geht [nicht] um die Bereitstellung von Kampftruppen am Boden.” Siehe Plenarprotokoll 14/198, S. 19825, vgl. Phillip Scherrer, a.a.O. (Fn. 7), S. 255.

154 Siehe dazu mit Nachweisen auf Berichterstattung ebenda, S. 253 ff.

155 Siehe dazu etwa die Kleine Anfrage der Fraktion von Bündnis 90/Die Grünen, BT-Drs. 17/2757 bezüglich der Operationen verschiedener Task Forces in Afghanistan.

156 Vgl. Georg Axer, a.a.O. (Fn. 141), S. 82; Timo Noetzel / Benjamin Schreer, a.a.O. (Fn. 141), S. 16 . 
Ausnahme von den Abläufen im Regelfall ist im ParlBG jedoch nicht vorgesehen. ${ }^{157}$ Diese Praxis wird weithin als unzureichend kritisiert, und zwar nicht nur von den Oppositions- ${ }^{158}$, sondern auch von Regierungsfraktionen. ${ }^{159}$ Zudem stellt die Praxis einen Widerspruch zu $\$ 4$ ParlBG dar: Wenn bereits in den dort genannten Fällen geringer Bedeutung das Parlament (durch das vereinfachte Zustimmungsverfahren) beteiligt werden muss, sollte dies in erhöhtem Maße für die politisch umstrittene Mitwirkung an Kampfhandlungen gelten. Der Eindruck einer unzureichenden Beteiligung relativiert sich allerdings, wenn man den Vergleich zur britischen Regelung der Draft Resolution zieht.

Spezialkräfte sind im Vereinigten Königreich noch stärker als in Deutschland aus dem Blick der Öffentlichkeit ferngehalten. Dementsprechend sieht s. 4 der Draft Resolution eine vollständige Ausnahme der Spezialkräfte vom gesamten Beteiligungsprozess vor. Dies umfasst nicht nur die Spezialkräfte selbst (s. 4. ss. 1a), die mit einer Stärke von etwa 2.000 Personen ${ }^{160}$ über beachtliche eigene Fähigkeiten verfügen, sondern auch sämtliche Angehörigen der regulären Streitkräfte, sofern sie lediglich die Spezialkräfte direkt oder indirekt unterstützen (s. 4. ss. 1b). Damit ist es der Regierung möglich, Einsätze der Spezialkräfte von einiger Größe vollständig ohne jede Beteiligung oder Information des Parlaments durchzuführen. Auch in dieser Frage sieht damit der deutsche Mechanismus (trotz seiner beschriebenen Unzulänglichkeiten) deutlich mehr Rechte für das Parlament vor.

\section{Erklärungsansätze und Ausblick}

Zusammenfassend lässt sich festhalten, dass die Parlamentsbeteiligung in Deutschland in sämtlichen Bereichen stärker ausgeprägt ist als im Vereinigten Königreich. Durch die Herleitung aus dem Grundgesetz kann sie zunächst vorrangige Geltung beanspruchen und ist gerichtlich voll durchsetzbar. Die Verwendung des Begriffs „Einsatz” anstelle von „armed conflict" als Auslöser sieht zudem eine Mitwirkung des Parlaments bei deutlich mehr militärischen Handlungsformen als nur dem umgangssprachlichen „Krieg” vor. In zeitlicher Hinsicht erlauben die nachträgliche Befassung und das Rückrufrecht eine ständige Beteiligung, während in der britischen Regelung nur ein fester Zeitpunkt vorgesehen ist (und dieser auch nur im Regelfall, im Eilfall etwa fällt die Beteiligung gänzlich weg). Auch gewährt die britische Regelung dem Premierminister an vielen Stellen ein Ermessen, etwa bezüglich des Vorliegens der Ausnahmefälle oder des Umfangs der an das Parlament geleiteten Informationen über den Einsatz. Zudem lässt die Draft Resolution der Regierung völlig freie Hand, Einsätze durch Spezialkräfte durchführen zu lassen und nimmt diese von jeglicher Parlamentsbeteiligung aus.

157 Vgl. Georg Axer, a.a.O. (Fn. 141), S. 82; ähnlich Volker Epping, a.a.O. (Fn. 89), Art. 87a Rn. 29.5; Andreas Gilch, a.a.O. (Fn 148), S. 220; Wolfgang Weiß, a.a.O. (Fn. 2), S. 109; auch fordert etwa die Fraktion Die Linke „die uneingeschränkte Umsetzung des Parlamentsbeteiligungsgesetzes" bezüglich des KSK, Minderheitenvotum der Fraktion Die Linke in der Beschlussempfehlung und Bericht des Verteidigungsausschusses, a.a.O. (Fn. 134), S. 164.

158 Siehe etwa die Anträge der Fraktionen Die Linke vom 10. Oktober 2007, BT-Drs. 16/6646, S. 2; Bündnis 90/Die Grünen vom 23. Oktober 2007, BT-Drs. 16/6770, S. 5 f.; Georg Axer, a.a.O. (Fn. 141), S. 82; Timo Noetzel / Benjamin Schreer, a.a.O. (Fn. 141), S. 15 f.

$159 \mathrm{Vgl}$. Beschlussempfehlung und Bericht des Verteidigungsausschusses, a.a.O. (Fn. 134), S. 135 f.

160 Vgl. Timo Noetzel / Benjamin Schreer, a.a.O. (Fn. 141), S. 16. 
Für diese generell exekutivfreundlichere Haltung im Vereinigten Königreich lassen sich verschiedene Gründe anführen. Strukturell mag es zunächst erstaunen, dass sich das britische Parlament trotz uneingeschränkter Regelungsbefugnis (wie oben beschrieben gibt es keinen verfassungsrechtlichen Schutz eines exekutiven Kernbereichs) nur sehr eingeschränkte Mitentscheidungsbefugnisse gegeben hat. Die liegt aber an der starken Stellung der Regierung im Parlament durch das Mehrheitswahlrecht. Eine wesentliche Reform bedürfte deshalb eines größeren Ansatzes. ${ }^{161}$ Demgegenüber ist die Kontrolle in Systemen mit Verhältniswahlrecht durch die Notwendigkeit häufiger Koalitionsbildung und die stärkere Stellung kleinerer Parteien deutlich ausgeprägter ${ }^{162}$, so auch in Deutschland. Ideologisch ist im Vereinigten Königreich der starke Einfluss der Ideen John Lockes zu „prerogative“ und „necessity" spürbar, dessen Argumentation sich noch heute in aktuellen Urteilen und wissenschaftlichen Beiträgen wiederfindet.

Wesentlichen Einfluss auf die gesellschaftliche Einstellung zum Streitkräfteeinsatz und damit auf die diesbezügliche Rechtslage hatte die Erfahrung der beiden Staaten mit Krieg. So kann man die Zurückhaltung der deutschen Wehrverfassung als Folge der „traumatisierten Nachkriegsgesellschaft” 163 verstehen: „Für die Nachkriegsdeutschen blieb alles, was nach Militär, Waffen und Gewalt aussah, gefährlich, unmoralisch und verächtlich."164 Dies ist typisch: Staaten, die militärische Niederlagen erfahren haben, sind Kampf gegenüber generell skeptischer. ${ }^{165}$ Dem steht in Großbritannien historisch ein großes Vertrauen in die Streitkräfte gegenüber - auch bereits zu Zeiten, in denen in Deutschland die Skepsis noch deutlich höher war als derzeit. ${ }^{166}$ Zudem wird dem Streitkräfteeinsatz in Großbritannien ein positives einigendes Moment zugeschrieben. ${ }^{167}$ Die Aufstellung als Berufsarmee führt weiterhin dazu, dass Verluste nur kleine Teile der Gesellschaft direkt betreffen und als Kon-

161 Vgl. David Jenkins, a.a.O. (Fn. 2), S. 162: „An intellectually honest approach to war prerogative reform in the UK requires that politicians and other commentators openly confront the elephant in the room'; that is, that the system of responsible government - as it has evolved under the strict party system - arguably now allows the government to control Parliament rather than the other way around." Vgl. Andrew Blick / Iain Byrne / Stuart Weir, a.a.O. (Fn. 44), S. 409; Samuel Issacharoff, a.a.O. (Fn. 43), S. 206 f.; David Jenkins, a.a.O. (Fn. 41), S. 260, S. 279; Keith Syrett, a.a.O. (Fn. 65), S. 118 f., S. 124.

162 Vgl. Thomas Risse-Kappen, Public Opinion, Domestic Structure, and Foreign Policy in Liberal Democracies, in: World Politics, 43. Jg. (1991), H. 4, S. 479 - 512, S. 488.

163 Otto Depenheuer, Der verfassungsrechtliche Verteidigungsauftrag der Bundeswehr - Grundfragen des Außeneinsatzes deutscher Streitkräfte, in: Deutsches Verwaltungsblatt, 102. Jg. (1997), H. 11, S. $685-689$, S. 685.

164 Peter Bender, Deutsche Außenpolitik: Vernunft und Schwäche, in: APuZ B 43/2008, S. 3 - 5, S. 4.

165 Vgl. Ola Listhang, War and Defence Attitudes: A First Look at Survey Data from 14 Countries, in: Journal of Peace Research, 23. Jg. (1986), H. 1, S. $69-76$, S. 73.

166 Vgl. ebenda, S. 75; Thomas Risse-Kappen, a.a.O. (Fn. 162), S. 497. Inzwischen nimmt zwar das Vertrauen in die Streitkräfte und die Zustimmung zu diesen in Deutschland ständig zu, jedoch gilt die Zustimmung eher der Verteidigung als den Auslandseinsätzen, siehe Gerhard Kümmel, The Winds of Change: The Transition from Armed Forces for Peace to New Missions for the Bundeswehr and Its Impact on Civil-Military Relations, in: Journal of Strategic Studies, 26. Jg. (2003), H. 2, S. 7 - 28, S. 17 f.

167 Vgl. Ulf Hedetoft, National Identity and Mentalities of War in Three EC Countries, in: Journal of Peace Research, 30. Jg. (1993), H. 3, S. 281 - 300, S. 284 f. 
sequenz der freiwilligen Verpflichtung gesehen werden. ${ }^{168}$ Demgegenüber war die Bundeswehr durch die Wehrpflicht bisher stärker in der Gesellschaft verankert.

Auch die Kolonialgeschichte spielt eine wesentliche Rolle. Im Gegensatz zu Deutschland hat das Vereinigte Königreich durch seine Kolonien eine lange und relativ ungebrochene Tradition des Auslandseinsatzes und der Stationierung von Streitkräften im Ausland. ${ }^{169}$ So fand auch die überwiegende Mehrzahl der Einsätze nach 1945 in Staaten des ehemaligen Empire statt. ${ }^{170}$ Auch dies ist typisch: Staaten mit Kolonialvergangenheit haben vielfach die Strukturen zum militärischen Eingreifen im Ausland beibehalten. ${ }^{171}$

Auch das Alter des Staats an sich kann das Verlangen nach demokratischer Kontrolle beeinflussen. So haben junge Demokratien tendenziell mehr parlamentarische Kontrolle, weil keine gesicherte Erfahrung mit gleichartigen Entscheidungen vorliegt und damit das zukünftige Verhalten der Regierung weniger vorhersehbar ist. ${ }^{172}$ Insofern kann das Vereinigte Königreich auf eine lange und vergleichsweise bruchlose Geschichte zurückschauen.

Ein weiterer Faktor ist die Einordnung der Frage in politische und rechtliche Systeme. Während das Bundesverfassungsgericht gesellschaftlich geduldet ${ }^{173}$ politische Fragestellungen in den rechtlichen Rahmen übernimmt, wird die Frage des Streitkräfteeinsatzes im Vereinigten Königreich nicht nur von Gerichten als für das rechtliche Verfahren ungeeignet angesehen. Entsprechend große Spielräume werden den politischen Akteuren zugestanden.

Die aufgezeigten Ursachen für die sehr unterschiedliche Rolle der Parlamente beim Auslandseinsatz deutscher und britischer Streitkräfte haben tiefe Wurzeln in verfassungsrechtlichen Grundentscheidungen, in Ideologie und Geschichte der Staaten. Vor diesem Hintergrund ist der Forderung, eine gemeinsame Europäische Armee müsse „ein Parlamentsheer werden"174, mit einiger zu Vorsicht begegnen: Der Weg zu einer gemeinsamen europäischen Position dürfte lang und steinig werden. Denkbarer Kompromiss - und eine Verbesserung gegenüber den beschriebenen Unzulänglichkeiten beider Systeme - wäre ein Ausschussmodell. So könnte durch den kleineren Personenkreis sowohl dem Bedürfnis nach Geheimhaltung und Geschwindigkeit Sorge getragen als auch eine umfassendere Information und Mitentscheidung eines direkter demokratisch legitimierten Organs ermöglicht werden.

168 Vgl. Hew Strachan, The Civil-Military, Gap' in Britain, in: Journal of Strategic Studies, 26. Jg. (2003), H. 2, S. 43 - 63, S. 51.

169 Vgl. ebenda, S. 44; Wolfgang Wagner, Parliamentary Control of Military Missions: Accounting for Pluralism, DCAF Occasional Paper Nr. 12, Genf 2006, http:/www.agora-parl.org/sites/default/files/parliamentary_control_of_military_missions.pdf (Abruf am 29. Mai 2011), S. 30.

170 Vgl. John Van Wingen / Herbert Tillema, a.a.O. (Fn. 3), S. 294 f.

171 Vgl. WEU Assembly, A/1762, Paris 2001, http://www.assembly-weu.org/en/documents/sessions_ordinaires/rpt/2001/1762.pdf (Abruf am 29. Mai 2011), S. 6.

172 Vgl. Wolfgang Wagner, a.a.O. (Fn. 169), S. 30.

173 Eine große Mehrheit der Bürger ist mit der Aufgabenerfüllung des Bundesverfassungsgerichts zufrieden, siehe Rüdiger Fiebig / Carsten Pietsch, a.a.O. (Fn. 100), S. 38 f.

174 Vgl. Andreas Fischer-Lescano / Timo Tohidipur, Rechtsrahmen der Maßnahmen gegen die Seepiraterie, in: Neue Juristische Wochenschrift, 62. Jg. (2009), H. 18, S. 1243 - 1246, S. 1244. 